\title{
ROBUST DESIGN OF A LENS SYSTEM OF VARIABLE REFRACTION POWER WITH RESPECT TO THE ASSEMBLY PROCESS
}

\author{
Ingo Sieber, Ulrich Gengenbach, Rudolf Scharnowell \\ Institute for Applied Computer Science \\ Forschungszentrum Karlsruhe GmbH, Germany \\ Ingo.Sieber@iai.fzk.de
}

\begin{abstract}
The aim of this paper is to show that a compensation of manufacturing tolerances by means of the functional design is reasonable. The approach is discussed exemplarily in the application of a lens system of variable refraction power considering the assembly tolerances. This approach is based on the steps sensitivity analysis, tolerance analysis, and design optimisation and will result in a robust design with respect to the assembly process.
\end{abstract}

\section{Introduction}

A monolithic setup of optical systems is not possible, as this would give rise to topological and geometric problems. In addition, a uniform material system is lacking. The modular setup of these hybrid systems results in an isolated manufacture of the individual components and their later assembly in a single system. An important aspect of construction is to ensure a certain functionality of the combined system, which is closely linked with the geometry of the structure and the application conditions. To maintain the overall function of a system under the given manufacturing conditions, the system design has to be robust with respect to the expected tolerances. Hence, simulation and optimisation of hybrid systems as a function of the tolerances induced by the assembly process play a crucial role. The concept presented in this paper is the compensation of tolerance effects induced by the assembly process by means of the functional design (robust design).

\subsection{Application example}

By way of example the approach described above will be applied to the design of a functional model of an intelligent implant to recover the accommodation ability of the human eye. The ability to focus on a close-by object (accommodation) gets lost while ageing due to a stiffening of the human lens. A possible approach to recovering this ability is an intelligent implant on the basis of a mechatronic system, the so-called Artificial Accommodation system [1]. This system is built up of the

Please use the following format when citing this chapter:

Sicber, I., Gengenbach, U., Schamowell, R, 2008, in IFIP International Federation for Information Processing, Volume 260, Micro-Assembly Technologies and Applications, eds. Ratchev, S., Koelemeijer, S., (Boston: Springer), pp. 87-93. 
functional units of signal acquisition, signal processing, active optics, control, and energy supply. The presented work addresses the compensation of tolerances induced in the optical system by the assembly process. The optical system is very sensitive with respect to tolerances, since small position errors will already result in noticeable degradation of the performance of the total system.

The active optical system consists of a lens system which varies its refraction power by means of a lateral movement of one of its components and an actor which performs the movement in a defined manner.

As an optical element, a so-called Alvarez-Humphrey lens [2] is used (Fig. 1).
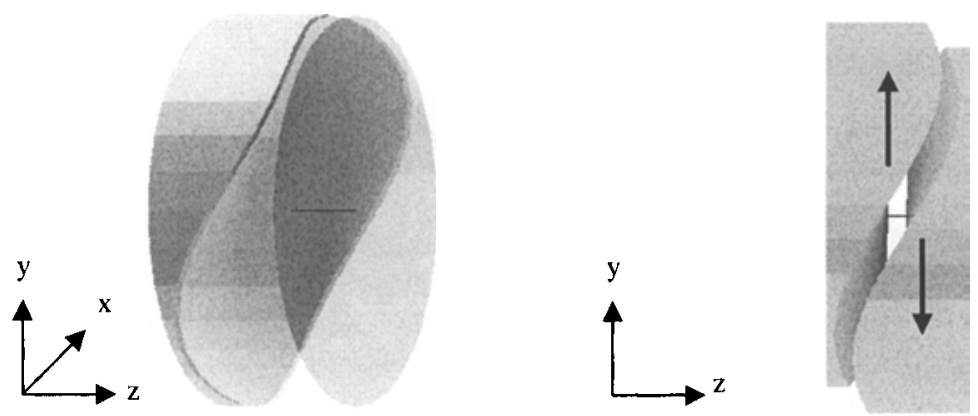

Fig. 1. Functioning of an Alvarez-Humphrey optics with a variable refraction power.

The Alvarez-Humphrey lens consists of two parts, the opposing surfaces of which are conjugated. The surface sag of the opposing surfaces is given by equation (1) with the lateral movement $v$ and the parameter $A$ :

$$
z(x, y)=A\left((y-v) x^{2}+1 / 3(y-v)^{3}\right)
$$

The relation between the lateral movement $\mathrm{v}$ and the change in refraction power $\Delta \mathrm{D}$ is given by equation (2):

$\triangle \mathrm{D} \sim \mathrm{A} v$

The functional model of the artificial accommodation system among others serves to demonstrate the functioning of the total system and the interaction between the individual components. To reach this goal, it is not necessary to keep the dimensions, but it is allowed to scale the system. The functional model of the implant is designed to fit in an eye model the size of a grapefruit. The simulation model of the optical subsystem is shown in Fig. 2. 


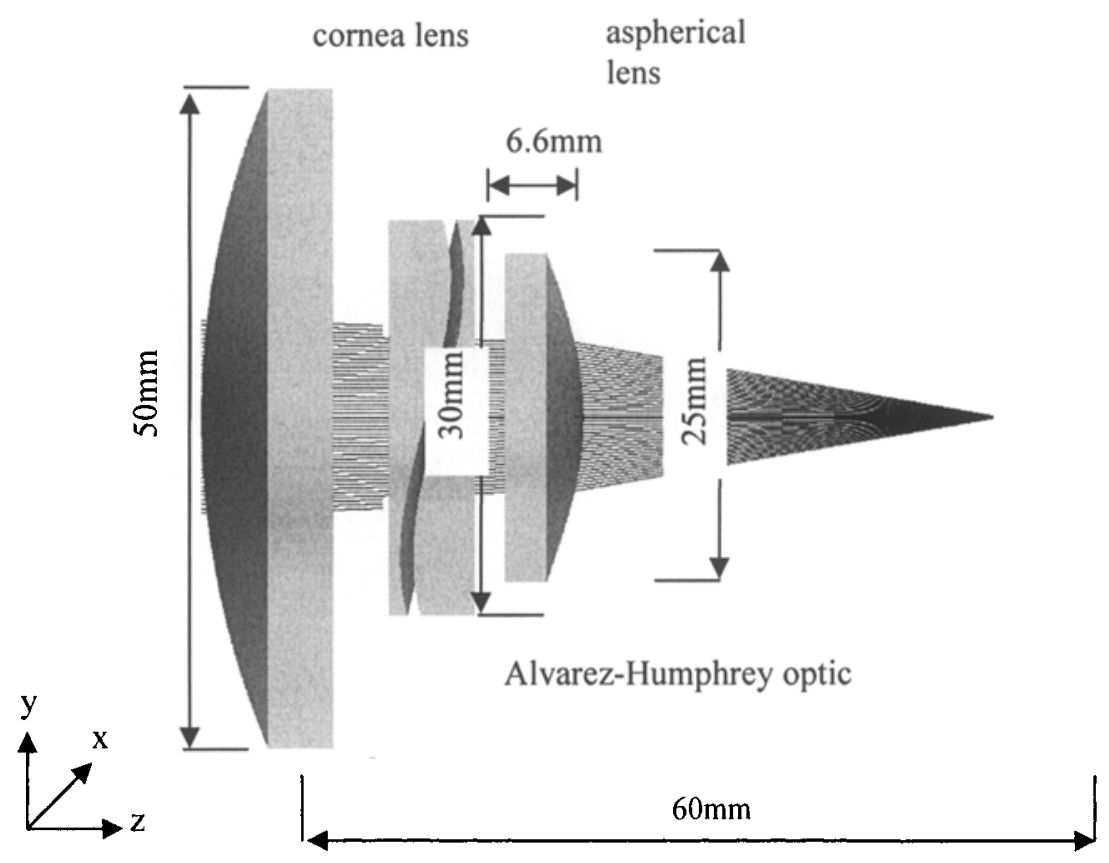

Fig. 2. Simulation model of the optical subsystem of the eye model.

\section{Assembly tolerances}

Optical refraction power is varied by means of a lateral movement of the first Alvarez-Humphrey surface in y-direction. To yield a good optical performance, both Alvarez-Humphrey surfaces must be well adjusted. An error in positioning these two parts may have a severe impact on the performance of the total system. Consequently, the most interesting part for a tolerance analysis of the optical subsystem is the Alvarez-Humphrey optics.

Three main types of assembly tolerances may be identified:

- lateral position error in x-direction (Fig. 3, left)

- wedge error (Fig. 3, middle)

- orientation error (Fig. 3, right) 

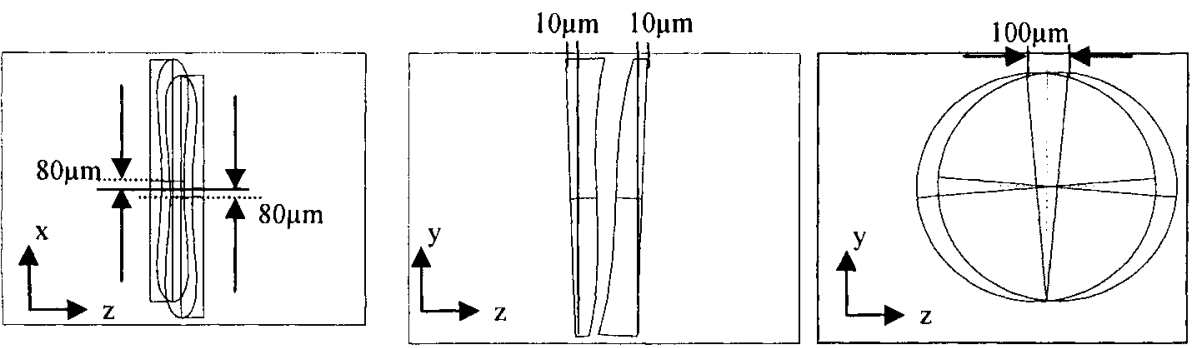

Fig. 3. Different types of assembly tolerances identified for the Alvarez-Humphrey optics.

\section{Tolerance analysis and optimisation}

One main feature of a robust design is that the difference in the performance criterion is minimised over the total tolerance range. This approach results in a design which ensures a defined functionality with a minimum deviation of performance over the total tolerance range.

As a criterion for the evaluation of the system's performance, the modulation transfer function (MTF) is chosen. The MTF, also known as spatial frequency response, is a direct measure of how well the various details in the object are reproduced in the image. The MTF is well-established to specify and judge the optical imaging quality [3].

The first step in tolerance analysis is a so-called sensitivity analysis. The sensitivity analysis is used to investigate the influence of the individual tolerances on the evaluation criterion. Hence, the worst-case value of the individual tolerance is chosen and the MTF is calculated. The simulations were carried out using the optical simulation tool ZEMAX-EE [4]. Fig. 4 depicts the MTF versus the spatial frequency for the three different types of assembly tolerances identified and the reference (from top left to bottom right: reference, lateral position error, wedge error, orientation error).

Comparison of the curves of the assembly tolerances with the reference curve leads to the following statements:

- the lateral position error has the most severe influence on the MTF. A breakdown of the curve below 25 cycles $/ \mathrm{mm}$ shows the decrease in the system's resolution due to this tolerance

- within the estimated tolerance range, the wedge error has no influence on the imaging quality of the system 
- the orientation error has a minor effect on the system's performance. Apart from the angular fraction, this tolerance also contains a fraction of lateral displacement in $x$-direction. This lateral fraction causes the degradation in the trend of the MTF
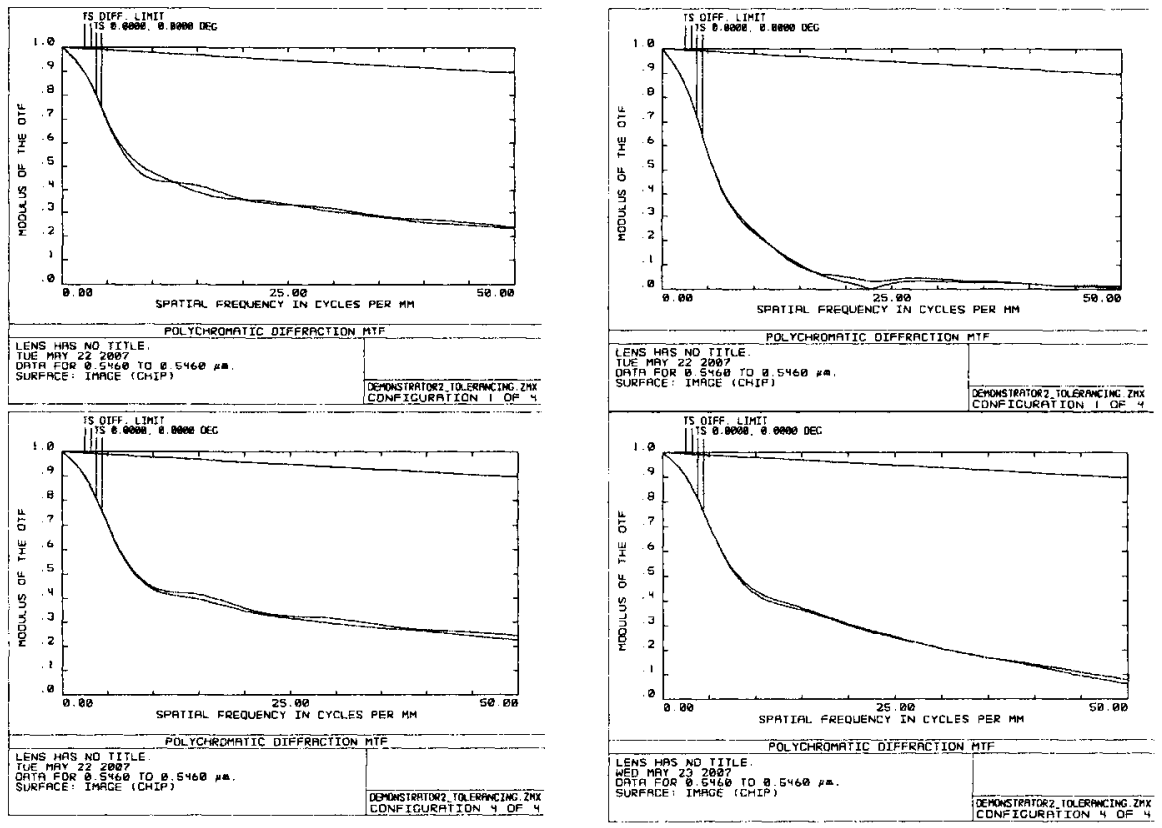

Fig. 4. MTF versus spatial frequency for the three different types of assembly tolerances identified and the reference (from top left to bottom right: reference, lateral position error, wedge error, orientation error).

The lateral position error is identified to be the tolerance with the most severe influence on the system's performance. On the basis of this position error a tolerance analysis is performed taking the estimated tolerance range into account.

To analyse the impact of the lateral position tolerance on the system's performance, a Monte Carlo analysis was performed. In a Monte Carlo analysis for a given tolerance range the change of the criterion is determined. For this purpose, the parameters having specified tolerances are set randomly using their defined range and a statistical model of the distribution of the parameters over the specified range. The statistical model used follows a normal distribution with a total width of four standard deviations between the extreme minimum and maximum allowed values. As the criterion, the MTF value at $12.5 \mathrm{cycles} / \mathrm{mm}$ is used. The results of 500 Monte Carlo runs are shown in Table 1: 


\begin{tabular}{|ll|}
\hline Nominal & 0.43242355 \\
Best & 0.43242698 \\
Worst & 0.22679682 \\
Mean & 0.40122752 \\
Std Dev & 0.03666711 \\
$98 \%<=$ & 0.43238019 \\
$90 \%<=$ & 0.43199561 \\
$50 \%<=$ & 0.41444874 \\
$10 \%<=$ & 0.35321565 \\
$2 \%<=$ & 0.26861138 \\
\hline
\end{tabular}

Table 1. Result of 500 Monte Carlo runs for the original design. The criterion is the MTF@12.5cyc./mm.

Under the assumption of a normal distribution of the tolerances, the MTF of the system is worse than 0.353 at 12.5 cycles per $\mathrm{mm}$ with a likelihood of $10 \%$. The value of the standard deviation also is of interest. This parameter provides information on the oscillation of the system's performance within the tolerance range.

\section{I Optimisation with respect to a robust design}

On the basis of the tolerance analysis an optimisation is carried out, the goal being to achieve a robust design. As before in the tolerance analysis, again the MTF value at 12.5 cycles per $\mathrm{mm}$ is chosen as the main criterion. The parameters of the optimisation are the coefficient A of the Alvarez-Humphrey surfaces (equation (1)) and higher-order coefficients of the polynomial equation of the surface sag (see equation (3)):

$z=a_{1} x^{2} y+a_{2} y^{3}+a_{3} x^{3} y+a_{4} y^{4} a_{5} x^{4} y$

The method used for optimisation is an actively damped least squares algorithm provided by the optical simulation tool ZEMAX-EE.

\section{Results and conclusions}

The optimised coefficients of the active lens serve as input for another Monte Carlo analysis. The results of this analysis allow for a comparison of the impacts of the tolerances on the system's performance between the original and the optimised design.

Table 2 shows the comparison of the results. The data of the original design are depicted on the left hand side, the data of the optimised design on the right. 


\begin{tabular}{|ll|ll|}
\hline \multicolumn{2}{|l|}{ Original design } & \multicolumn{2}{|c|}{ Optimised design } \\
\hline Nominal & 0.43242355 & Nominal & 0.45563401 \\
Best & 0.43242698 & Best & 0.45582751 \\
Worst & 0.22679682 & Worst & 0.30953146 \\
Mean & 0.40122752 & Mean & 0.43430906 \\
Std Dev & 0.03666711 & Std Dev & 0.02611409 \\
$98 \%<=$ & 0.43238019 & $98 \%<=$ & 0.45567880 \\
$90 \%<=$ & 0.43199561 & $90 \%<=$ & 0.45527413 \\
$50 \%<=$ & 0.41444874 & $50 \%<=0.44379105$ \\
$10 \%<=$ & 0.35321565 & $10 \%<=0.39642523$ \\
$2 \%<=$ & 0.26861138 & $2 \%<=$ & 0.35649227 \\
\hline
\end{tabular}

Table 2. Comparison of the results of 500 Monte Carlo runs for the original and the optimised design. The criterion is the MTF@12.5cyc./mm.

An analysis of the optimisation with respect to tolerances yields the following results:

- a slight enhancement of the nominal value (about $5.4 \%$ ), caused by the addition of further coefficients in the surface description.

- enhancement of the worst value by $40.0 \%$

- enhancement of the mean value by $10.7 \%$

- enhancement of the standard deviation by $40.1 \%$

- looking again at the MTF value of 0.353: as seen, using the original design the performance of the system is worse than that value with a likelihood of $10 \%$. Using the optimised system the likelihood of being worse in performance shrinks to $2 \%$.

The results show clearly that a compensation of influences caused by manufacturing tolerances is reasonable by means of the functional design. The approach was derived and applied to compensate for assembly tolerances by means of the functional design of an Alvarez Humphrey optic. This approach is based on the following steps:

- sensitivity analysis to identify the worst offenders

- tolerance analysis on the basis of the Monte Carlo method

- design optimisation of the functional components

The result is a robust design with respect to the assembly process.

\section{References}

1. U. Gengenbach, G. Bretthauer, and R. F. Guthof, Kunstliches Akkommodationssystem auf der Basis von Mikro- und Nanotechnologie. Mikrosystemtechnik Kongress, Freiburg. VDE Verlag Berlin Offenbach (2005) 411-414

2. L. W. Alvarez, and W. E. Humphrey, Variable power lens and system. US Patent 3507565 (1970)

3. G. H. Smith: Practical Computer-Aided Lens Design, Willmann-Bell, Inc. 1998

4. ZEMAX: Software For Optical System Design, http://www.zemax.com 